\title{
Analysis on the Effect of the Nonlinear Resistance on the Locomotive Operating Overvoltages
}

\author{
Zhengqing Han, Donglin Zhang, Yuning Wu, Shibin Gao \\ School of Electrical Engineering, Southwest Jiaotong University, Chengdu, China
}

Received February, 2013

\begin{abstract}
With the application of the articulated phase insulator, and the speed of electric locomotive rising, it is inevitable for the electric locomotive to adopt the technology automatic passing through the electric phase separation. However, when the locomotive passes the electric phase separation, a variety of overvoltages will be generated, such as the cut-off overvoltage and the closing overvoltage. In this paper, the causes of the two overvoltages above are analyzed theoretically and simulated in Simulink. Then this paper discusses the suppression effects on the cut-off overvoltage and the closing overvoltage by paralleling the nonlinear resistance and the main breaker, or parallelling the nonlinear resistance and the locomotive transformer. The simulation results show that parallelling the nonlinear resistance and the locomotive transformer has suppressive effects on the two overvoltages mentioned above.
\end{abstract}

Keywords: Articulated Phase Insulator; Switching Overvoltage; Nonlinear Resistance; SIMULINK

\section{Introduction}

The application of the articulated phase insulator can eliminate the mechanical hard point problem caused by the device phase separation, and increase the reliability of the electric locomotive. With the speed rising, the time interval of passing through the phase separation is reduced. When the electric locomotive passes through the electric phase separation, the traditional approach relies on the driver's operation, demoting and closing auxiliary motor, and disconnecting main breakers in order. After the locomotive passes, it is then operated in the reverse order, so the pantograph can pass through phase separation in the non-current way, ensuring the service life of the catenary and pantograph [1]. The method is not adopted in the modern railway system because of its frequent operation and low safety factor, so it becomes a necessity to use the technology of automatic passing through the electric phase separation (including the vehicle auto-passing phase separation, column auto-passing phase separation, ground switching auto-passing phase separation [2]). When the locomotive passes automatically through the phase separation, the operation of main breaker will cause the cut-off overvoltage and the closing overvoltage, which are analyzed in literature [3-5]. As the generated overvoltage threatens the running safety of locomotive, it is important to find the corresponding suppressive measures to ensure the safe operation of the electric locomotive.

This paper analyzes the causes of the cut-off over- voltage and the closing overvoltage when passing through the phase separation with adopting the combination of the ground installations and the column switch. Literature [6] researched the RC components suppressor. Literature [1], [6] mentioned the suppression of nonlinear resistances, but both were with no simulations. This paper discusses the suppressive effects of the operating overvoltage by using Matlab.

\section{Electric Process Analysis}

\subsection{The Cut-off Overvoltage}

The Figure 1 that can found in [7] shows that the locomotive passes through the phase separation with the combination of the ground installations and the column switch.

When the electric locomotive passes point $\mathrm{A}$, the main breaker is automatically disconnected, with the huge current being cut off which generates arcs. If the interrupters are not strong enough, the electric locomotive will rush through the phase separation charged, leading

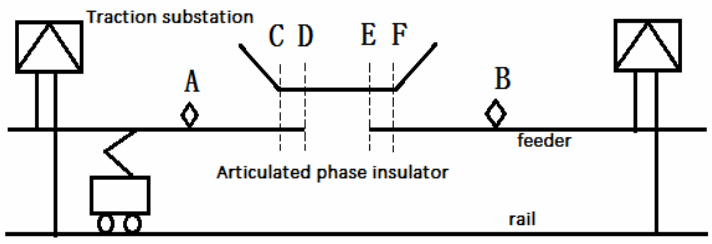

Figure 1. The schematic diagram of electric locomotive passing the articulated phase insulator. 
to the damage of the pantograph and catenary. In severe cases, it will result in the traction substation tripped accident. Otherwise, it will generate high cut-off overvoltage.

\subsection{The Closing Overvoltage}

After the locomotive passes through the phase separation to point $\mathrm{B}$, the breaker is required to be closing to resume power. This operation of the breaker may generate the closing overvoltage whose peak is related to the catenary voltage. And locomotive auxiliary winding and asynchronous auxiliary cluster are still in the working state. Some motors act as the electromotor, while others act as the generator, so the auxiliary systems can be seen as a power. The voltage of auxiliary, which is called the residual voltage, can be coupled to the primary side of the locomotive transformer, whose peak is related to the number of auxiliary motors. Due to the presence of residual voltage, the complete response of the closing circuit is the superposition of the zero-state response and the zero-input response, which may increase the peak of overvoltage, and even lead to the tripped accident of the traction substation.

\section{The Equivalent Circuit of the Overvoltage}

\subsection{The Equivalent Circuit of Cut-off Overvoltage}

According to the characteristics of the traction network that the line reactance is much bigger than the resistance, the intercepting overvoltage equivalent circuit is set up as shown in Figure 2. L1 is the sum of the equivalent reactance of the traction substation and the contact line. C1 is the circuit equivalent capacitance of the circuit ground. L2 is the locomotive's main circuit, while C2 is its equivalent capacitance to earth.

As seen in Figure 2 that can found in [1], an equation can be drawn as follows.

$$
C_{T} \frac{d u}{d t}+\frac{1}{L_{T}} \int u d t=0
$$

Namely,

$$
\frac{d^{2} u}{d t^{2}}+\frac{1}{L_{T} C_{T}} u=0
$$

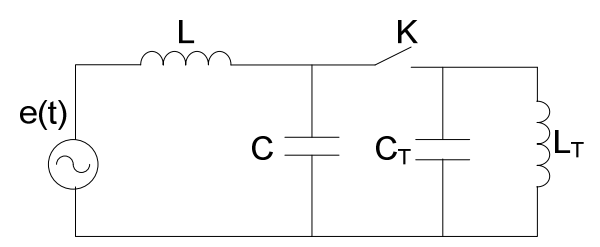

Figure 2. The equivalent circuit of the intercepting overvoltage.
Assuming that the two initial values are $u_{0}$ and $u_{0}^{\prime}$, the Equation (1) can be simplified by using Laplace transform.

$$
U(s)=\frac{s u_{0}+u_{0}^{\prime}}{s^{2}+\left(L_{T} C_{T}\right)^{-1}}
$$

Using the inverse transformation of Laplace to simplify the Equation (2),

$$
\begin{aligned}
u & =\frac{1}{2 j w_{0}}\left[j w_{0} u_{0}\left(e^{j w_{0} t}+e^{-j w_{0} t}\right)+u_{0}^{\prime}\left(e^{j w_{0} t}-e^{-j w_{0} t}\right)\right] \\
& =u_{0} \cos w_{0} t+\left(u_{0}^{\prime} \sin w_{0} t\right) / w_{0} \\
& =\sqrt{u_{0}^{2}+\left(u_{0}^{\prime} / w_{0}\right)^{2}} \sin \left(w_{0} t+\beta\right)
\end{aligned}
$$

where $w_{T}=\sqrt{1 / L_{T} C_{T}}$ and $\beta=\operatorname{arctg} \frac{u_{0} w_{0}}{u_{0}^{\prime}}$

When the current is cut off, it is known that $u_{0}=E_{m} \cos \alpha$ and $i_{0}=I_{m} \sin \alpha$. Then the conclusion can be drawn as both the inductive current and the capacitance voltage could not mutate.

$$
\left[\begin{array}{l}
u_{0} \\
u_{0}^{\prime} \\
I_{m} \\
f_{N}
\end{array}\right]=\left[\begin{array}{l}
E_{m} \cos \alpha \\
I_{m} \sin \alpha / C_{T} \\
E_{m} / 2 \pi f L_{T} \\
1 / 2 \pi \sqrt{L_{T} C_{T}}
\end{array}\right]
$$

$K_{m}$ is the ratio of intercepting overvoltage to the traction voltage.

$$
K_{m}=\frac{\sqrt{u_{0}^{2}+\left(u_{0}^{\prime} / w_{0}\right)^{2}}}{E_{m}}=\sqrt{\cos ^{2} \alpha+\left(\frac{f_{N}}{f}\right)^{2} \sin ^{2} \alpha}
$$

Because of $f_{N} \gg f, K_{m}$ can be approximately seen as

$$
K_{m}=\left(\frac{f_{N}}{f}\right) \sin \alpha
$$

From Equation (6), it could be obtained that the intercepting overvoltage is proportional to the circuit resonance frequency and the contact line when the current is cut down.

\subsection{The Equivalent Circuit of Closing Overvoltage}

After the locomotive passes through the phase separation, the circuit can be equivalent as shown in Figure $\mathbf{3}$ for the moment the switch is on. The parameters in Figure 3 [8] are basically the same with the ones in the Figure 2.

In Figure 3, $L_{2} \gg L_{1}$ and $C_{1}>>C_{2}$. Therefore, the influence of $L_{1}, C_{2}$ can be ignored, the differential equation can be listed as follows:

$$
R C \frac{d u}{d t}+u+\frac{R}{L_{T}} \int u d t=e(t)
$$




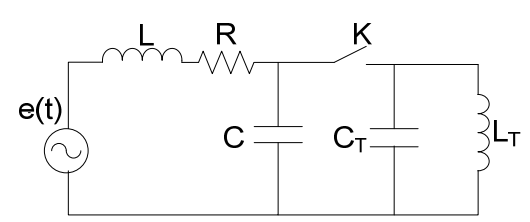

Figure 3. The equivalent circuit of the closing overvoltage.

$$
\text { Namely } \quad R C \frac{d^{2} u}{d t^{2}}+\frac{d u}{d t}+\frac{R}{L_{T}} u=e^{\prime}(t)
$$

Due to the effect of residual voltages, the formula above can be seen as a constant coefficient second order linear inhomogeneous differential equation, whose solution includes the zero-input response and zero-state response.

The zero-input response

The zero-input responses of the formula (8) is

$$
R C \frac{d^{2} u}{d t^{2}}+\frac{d u}{d t}+\frac{R}{L_{T}} u=0
$$

And the eigenvalues are

$$
P_{1,2}=-\frac{1}{2 R C} \pm \sqrt{\left(\frac{1}{2 R C}\right)^{2}-\frac{1}{L_{T} C}}
$$

The initial value can be defined as

$$
u_{0}=E_{f} \sin a \text { and } u_{0}=E_{f} \sin a .
$$

$E_{f}$ denotes the residual peak, the equation (9) can be simplified by using Laplace transform as follows:

$$
U(s)=\frac{R C S u_{0}+u_{0}}{R C S^{2}+S+R / L_{T}}
$$

The zero-state response of circuit is

$$
u_{x}=\frac{p_{2} u_{0}}{p_{2}-p_{1}} e^{p_{1} t}+\frac{p_{1} u_{0}}{p_{1}-p_{2}} e^{p_{2} t}
$$

The zero-state response

Defining $e(t)=E_{m} \sin (w t+\beta)$, the impulse response can be got with the zero-state response. The impulse response is got as follows:

$$
h(t)=\frac{1 / R C}{p_{1}-p_{2}}\left(e^{p_{1} t}-e^{p_{2} t}\right)
$$

The zero-state response is:

$$
u_{f}=\int_{0}^{t} f(\tau) h(t-\tau) d \tau
$$

The complete response of the system is the sum of both the zero-input response and the zero-state response.

\section{Simulation}

Based on the theoretical analysis, a large number of simulations are carried out by using the model in Simulink. The simulation parameters are as below: voltage of cate- nary $=27.5 \mathrm{kV}, \mathrm{L} 1=0.01749 \mathrm{H}, \mathrm{C} 1=1.85 \mathrm{e}-9 \mathrm{~F}, \mathrm{C} 2=$ 2.973e-10F, L2 = 10.1H.

\subsection{Simulation of the Cut-off Overvoltage}

Figure 4(a) shows high voltages will not be generated when disconnecting the main breaker while the voltage of catenary reaches 0 .

Figure 4(c) shows it will produce an overvoltage with the peak value reaching $129.29 \mathrm{kV}$ when disconnecting the main breaker while the voltage of catenary reaches its peak value. The value $129.9 \mathrm{kV}$ is 3.34 times of the normal peak value, and far beyond the regulated voltage level of the safe operation of the electric locomotive.

\subsection{Simulation of the Closing Overvoltage}

The closing operation needs to be done after the locomotive passed through the phase separation. Different peak values of the closing overvoltage will be generated when the catenary voltage is in different phases.

The simulation results Figure 5 (a) show that higher overvoltage will not be caused when the phase of the catenary voltage is around $0^{\circ}$ or $180^{\circ}$. While the phase of the catenary voltage is around $90^{\circ}$ or $180^{\circ}$, the Figure 5 (b), (c) show the peak value of the overvoltage reaches $74 \mathrm{kV}$, which is far beyond the regulated voltage level of the safe operation of the electric locomotive.

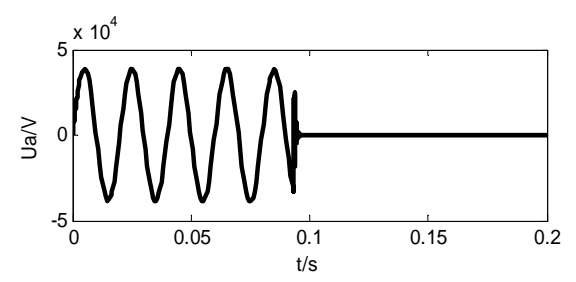

(a) The phase of the catenary voltage being $0^{\circ}$

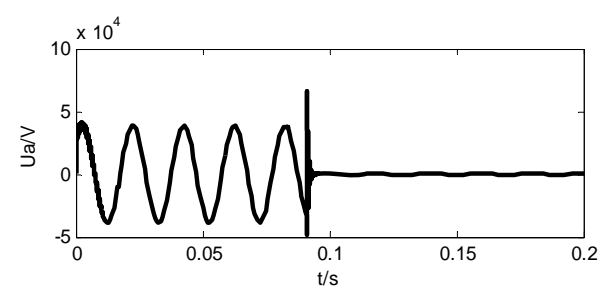

(b) The phase of the catenary voltage being $45^{\circ}$

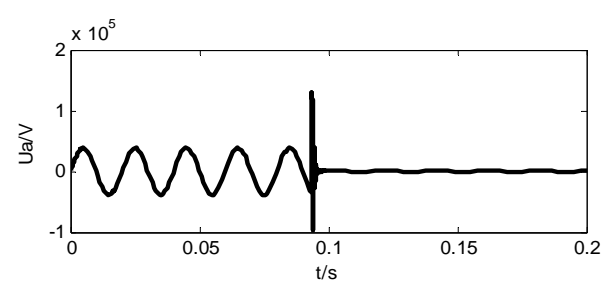

(c) The phase of the catenary voltage being $90^{\circ}$

Figure 4. The cut-off overvoltage caused by different phases of the catenary voltage. 


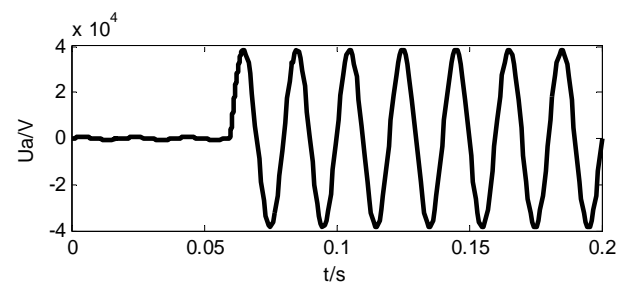

(a) The phase of the catenary voltage being $0^{\circ}$

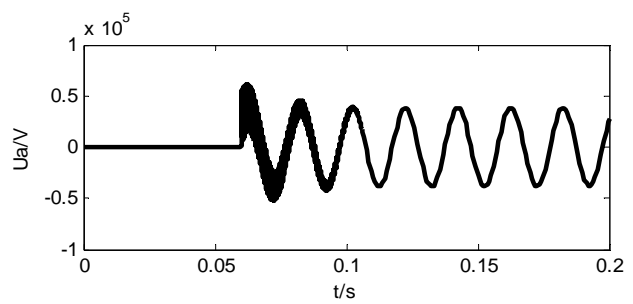

(b) The phase of the catenary voltage being $45^{\circ}$

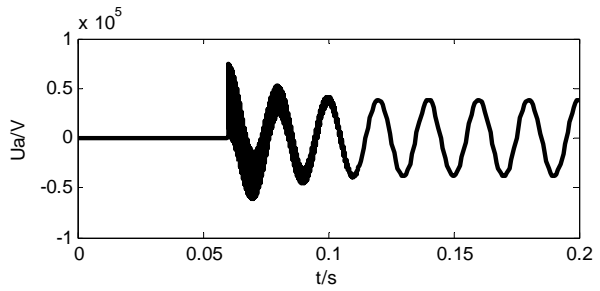

(c) The phase of the catenary voltage being $90^{\circ}$

Figure 5. The closing overvoltage caused by different phases of the catenary voltage.

\section{Suppression Measures}

\subsection{Model of the Nonlinear Resistance}

The overvoltage has a serious influence on the safe operation of the electric locomotive. The traditional demoting operation aims to decrease the levels of the overvoltages, but it is no longer suitable for the high-speed train, so better suppression measures involving hardwares need to be found.

Usually, in power systems, transient voltages caused by direct lightning strikes are prevented by installing arrester (MOA) [9]. The arrester itself is a kind of nonlinear resistance (MOV), composed of $\mathrm{ZnO}$ (the main ingredient) and a few other metal oxygen content additives that are used to constitute grain boundaries phase and improve some properties of the arrester [10]. If the device is specially processed to lower its voltage level, the arrester can be installed in electric locomotive to suppress the operating overvoltage. The following is the analysis of the arrester's characteristics and the suppression effects on the two overvoltages mentioned above.

According to the characteristics of the nonlinear resistance, its model is built by using Matlab/Simulink platform, and its features are verified through simulation. Figure 6(a) shows the structure of the nonlinear resistance model, including CCS (Controlled current source),
Fcn (User-defined function: $\mathrm{I} 0 *(\mathrm{u} / \mathrm{V} 0) \wedge$ alap), $\mathrm{V}$ meter (voltmeter), and a first-order transfer function [11]. Figure 6(b) shows the characteristics of the simulation circuit. Figures 6(c), (d) and (e) respectively show the voltage waveform, the current waveform and the volt-ampere characteristics.

Figure 6(e) presents the properties of nonlinear resistance when its terminal voltage changes. When the terminal voltage is small, its resistance value is infinity, and when its terminal voltage is up to a certain extent, its resistance value reduces to zero quickly. These properties are called as the clamping voltage effect which can be used to suppress the cut-off overvoltage and the closing overvoltage.

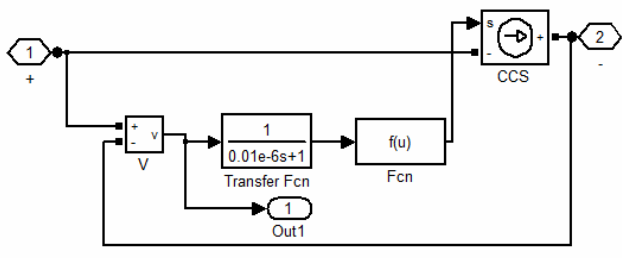

(a) The structure of the nonlinear resistance model

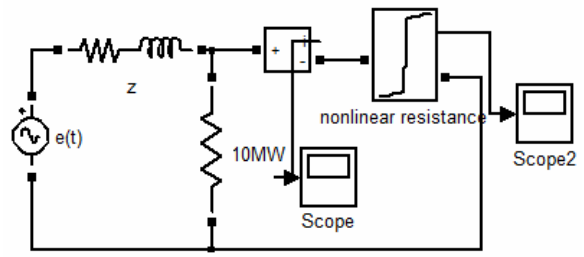

(b) The simulation circuit of the nonlinear resistance

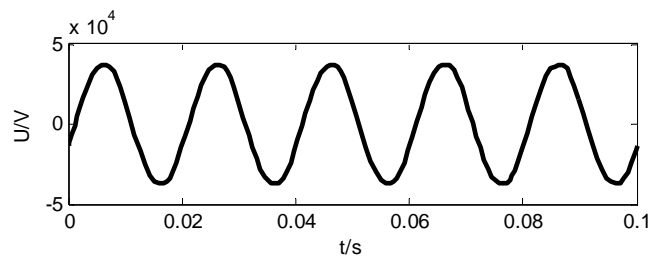

(c) The waveform of the voltage

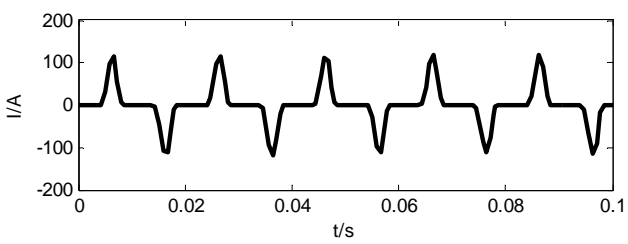

(d) The waveform of the current

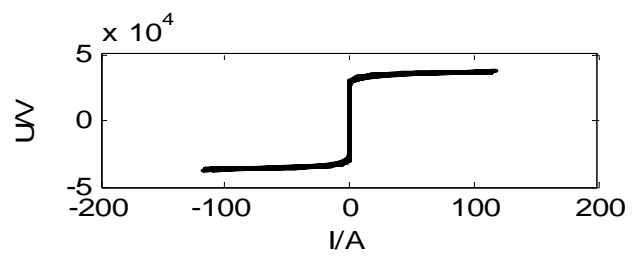

(e) The volt-ampere characteristics

Figure 6. Model and performances of the MOV. 


\subsection{Suppression Simulation of the Overvoltage}

Parallelling the nonlinear resistance and the main breaker, the suppressive effects on the two overvoltages are shown in Figure 7 (disconnecting or closing main breaker when the catenary voltage reaches its peak value).

When parallelling the nonlinear resistance and the main transformer of the locomotive, the suppressive effects on the two overvoltages are shown in Figure 8.

\section{Conclusions}

Through theoretical analysis and simulations, the conclusions can be drawn as follows:

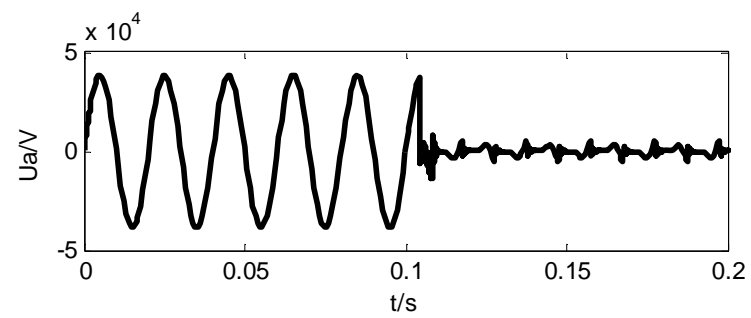

(a) The suppressive effects on the cut-off overvoltage

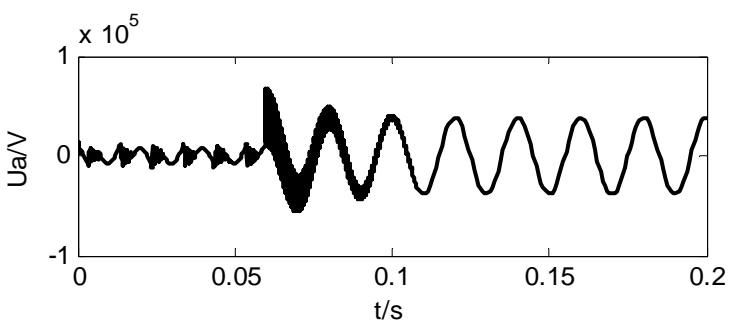

(b) The suppressive effects on the closing overvoltage

Figure 7. Parallelling the nonlinear resistance and the main breaker.

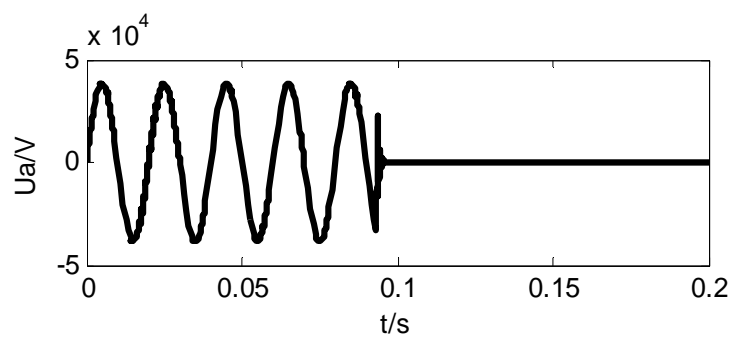

(a) The suppressive effects on the cut-off overvoltage

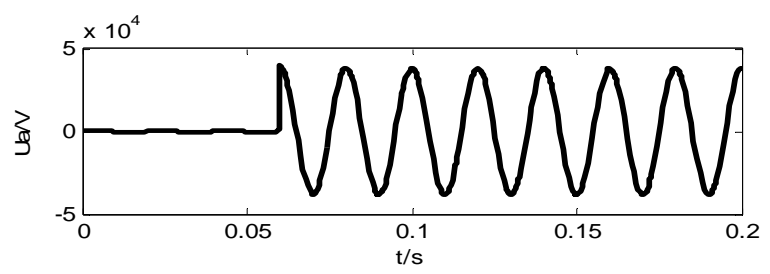

(b) The suppressive effects on the closing overvoltage

Figure 8. Parallelling the nonlinear resistance and the main transformer of the locomotive.
1) If the main circuit breaker is off when the phase of the catenary voltage is close to its peak phase, the cut-off overvoltage will be very high. Otherwise, if the main circuit breaker is on when the phase of the catenary voltage is close to its peak phase, the closing overvoltage will be very high.

2) The suppressive effects on the cut-off overvoltage and the switching overvoltage by placing the nonlinear resistances in different positions are obtained. When parallelling the nonlinear resistance and the main circuit breaker, the cut-off overvoltage can be inhibited efficiently, while the closing overvoltage cannot be inhibited; when parallelling the nonlinear resistance and the main transformer of the locomotive, both the cut-off overvoltage and the closing overvoltage can be inhibited efficiently.

\section{Acknowledgements}

The authors acknowledge the supports of the National Natural Science Foundation of China (Grant No. 50907055 and U1134205), the Sichuan Province Key Technology Research and Development Program of China (Grant No. 2011GZ0079) and the Fundamental Research Funds for the Central Universities (Grant No. SWJTU12CX028)

\section{REFERENCES}

[1] C. Z. Chen, "The Analysis on the Course of Electric Motive Passing though Phase Separation,” 2007, pp. 7-22.

[2] Y. S. Yan, "The Discussion on Electric Locomotive Auto-passing the Phase Separation," Electric Drive for Locomotive, Vol. 6, 1999, pp. 1-3.

[3] W. Ran, X. Li, B. Liu and Q. L. Zheng, "Research on Transient Process of Ground's Auto-passing Neutral Section at Switching Time," Transactions of China Electro Technical Society, Vol. 26, No. 11, 2011, pp. 150-154.

[4] X. J. Wei, H. T. Wang and Y. S. Wu, "Study on the Ground Automatic Passing the Articulated Phase Insulator with Parallel Resistance,” Electric Railway, Vol. 2, 2011, pp. 5-8.

[5] Y. N. Gu, Y. Wang and H. Wang, "The Analysis on Overvoltage of Electric Locomotive Automatic Passing Phase Separation,” Electric Railway, Vol. 3, 2009, pp. 1-3.

[6] P. F. Yin and W. C. Du, "Generation and Control of Cut-off over Voltage of a Vacuum Circuit Breaker," Electrical Switching, Vol. 2, 2005, pp. 34-37.

[7] Y. S. Gong, "Research of Over-voltages of Electric Locomotive Passing the Articulated Phase Insulator," Journal of the China Railway Society, Vol. 30, No. 4, 2008, pp. 103-107.

[8] W. G. Pan, "The Analyze on the Transient Process of Switch-Ground's Automatic Passing Phase-Separation Device for Traction Network of Electrified Railway," 
2008.

[9] Y. L. Wang, D. Y. Zhao and S. H. Hu, "Development Trends of Line Arresters,” Electrical Porcelain Lightning Arrester, Vol. 1, 2011, pp. 26-34.

[10] Y. J. Liang, “Application of Metal Oxide Varistors in
Power System,” Beijing, Vol. 5, 1997, pp. 74-90.

[11] J. Wang and G. Q. Weng, "MATLAB/SIMULINK Simulation and Application of Electric Power System," Xi'an, 2008, pp. 271-275. 